\title{
Influence of oval defects on transport properties in high-mobility two-dimensional electron gases
}

L. Bockhorn, A. Velieva, S. Hakim, T. Wagner, E. P. Rugeramigabo, D. Schuh, C. Reichl, W. Wegscheider, and R. J. Haug

Citation: Appl. Phys. Lett. 108, 092103 (2016); doi: 10.1063/1.4942886

View online: https://doi.org/10.1063/1.4942886

View Table of Contents: http://aip.scitation.org/toc/apl/108/9

Published by the American Institute of Physics

\section{Articles you may be interested in}

Improvement of the transport properties of a high-mobility electron system by intentional parallel conduction Applied Physics Letters 110, 042106 (2017); 10.1063/1.4975055

A complete laboratory for transport studies of electron-hole interactions in GaAs/AIGaAs ambipolar bilayers Applied Physics Letters 110, 072105 (2017); 10.1063/1.4976505

Electrically driven and electrically tunable quantum light sources

Applied Physics Letters 110, 071102 (2017); 10.1063/1.4976197

Profiling the local carrier concentration across a semiconductor quantum dot

Applied Physics Letters 106, 192101 (2015); 10.1063/1.4919919

MBE growth of high electron mobility 2DEGs in AIGaN/GaN heterostructures controlled by RHEED

AIP Advances 2, 012108 (2012); 10.1063/1.3679149

Interplay between quantum well width and interface roughness for electron transport mobility in GaAs quantum wells

Applied Physics Letters 109, 232105 (2016); 10.1063/1.4971824

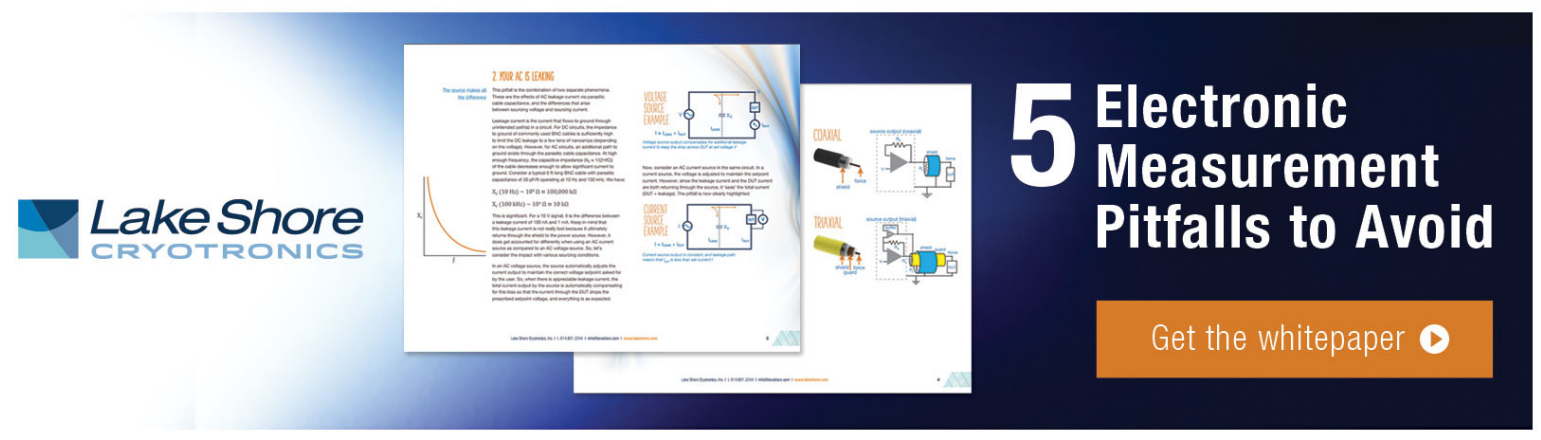




\title{
Influence of oval defects on transport properties in high-mobility two-dimensional electron gases
}

\author{
L. Bockhorn, ${ }^{1, a)}$ A. Velieva, ${ }^{1}$ S. Hakim, ${ }^{1}$ T. Wagner, ${ }^{1}$ E. P. Rugeramigabo, ${ }^{1}$ D. Schuh, ${ }^{2}$ \\ C. Reichl, ${ }^{3}$ W. Wegscheider, ${ }^{3}$ and R. J. Haug ${ }^{1}$ \\ ${ }^{1}$ Institut für Festkörperphysik, Leibniz Universität Hannover, Germany \\ ${ }^{2}$ Institut für Experimentelle und Angewandte Physik, Universität Regensburg, Germany \\ ${ }^{3}$ Laboratorium für Festkörperphysik, ETH Zürich, Switzerland
}

(Received 21 October 2015; accepted 16 February 2016; published online 1 March 2016)

\begin{abstract}
Rare macroscopic growth defects next to a two-dimensional electron gas influence transport properties and cause a negative magnetoresistance. On the basis of this, we show that the number of oval defects seen on the material surface is comparable with the density of macroscopic growth defects determined from the negative magnetoresistance. We examine several materials with different densities of oval defects $n_{S}$ which were grown in one cycle under the same conditions to verify our observations. Paradoxically, the material with the largest number of oval defects has also the highest electron mobility. @ 2016 AIP Publishing LLC. [http://dx.doi.org/10.1063/1.4942886]
\end{abstract}

For a while, the observation of new effects in the fractional Quantum Hall regime $(\mathrm{FQHE})^{1-3}$ comes along with increasing electron mobility $\mu$. However, over the years, it became apparent that a high-mobility two-dimensional electron gas (2DEG) not suffices the observation of fragile FQHE features and the mobility alone cannot serve as a reliable indication for the quality of a sample. According to Sarma et al..$^{5}$ in addition to the transport scattering time $\tau=$ $m^{*} \mu / e$ also the quantum relaxation time $\tau_{q}$ must be considered, which can be calculated from the magnitude of the Shubnikov-de Haas $(\mathrm{SdH})$ oscillations. ${ }^{6}$ One assumes that both times are sensitive to different types of disorder. The transport scattering time $\tau$ is dominated by short-range scattering events, while the quantum relaxation time $\tau_{q}$ depends also on long-range disorder.

Usually, the considered sources of disorder are unintentional background impurities, roughness at the GaAsAlGaAs interface, alloy disorder, random charged impurities at the GaAs-AlGaAs interface, and remote dopant impurities which give rise to long-range disorder (e.g., see Refs. 4 and $5)$. Here, we concentrate on a further source of disorder which is known for many years but is, in general, disregarded for transport in modern high-mobility 2DEGs: macroscopic growth defects. These macroscopic defects occur in small numbers anywhere in the material and can be seen as the so-called oval defects on the surface.

In the following, we study the influence of oval defects on the transport properties of high-mobility 2DEGs. Therefore, we examined several materials with clearly different numbers of oval defects on the material surface. We observe a clear influence on the low temperature magnetotransport measurements and show that scattering on oval defects becomes relevant in the sample with the highest mobility. Furthermore, we find with increasing number of oval defects a stronger manifestation of the negative magnetoresistances at small magnetic fields and determine the number of macroscopic defects also from Ref. 7.

\footnotetext{
a)bockhorn@nano.uni-hannover.de
}

Oval defects can be caused by many sources as shown in different proposals, e.g., Refs. 8-12. Their density and size strongly depend on the growth conditions. Our materials were grown by molecular beam epitaxy in one growth cycle. Hence, they were produced under identical conditions and have nearly the same layer structure. The main difference was the temperature at the orifice of the Ga crucible. To leave the Ga flow unchanged also the temperature at the bottom of the Ga crucible was adjusted. Correspondingly, more $\mathrm{Ga}$ condenses at the orifice for lower temperatures and forms droplets. These Ga droplets could be evaporated to the substrate, where they act as nucleation sites for unbounded Ga

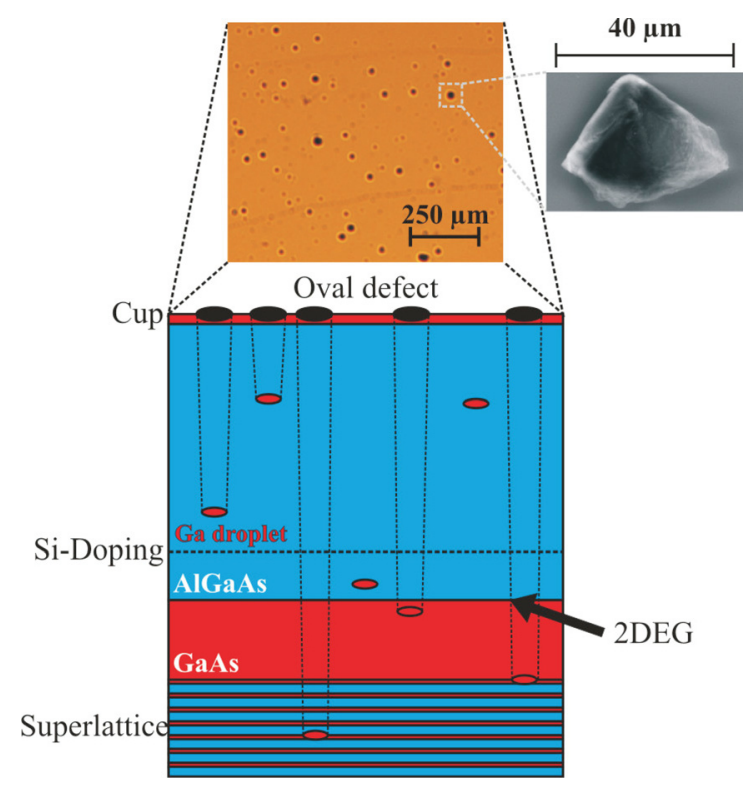

FIG. 1. Macroscopic defects are clearly visible on the unstructured material surface. These so-called oval defects are distributed inhomogeneously with varying size. A magnification of one oval defect is illustrated in an additional picture. Usually, the formation of oval defects is caused by Ga droplets anywhere in the material. The supposed Ga droplets are marked by red ovals in the schema of the layer structure. The layer schema also indicates that the examined 2DEG is realized in a heterojunction at the GaAs-AlGaAs interface. 
atoms and lead to macroscopic growth defects. In the crystal structure, holes arise due to these macroscopic growth defects. During the further growth process, the holes broaden, and they are finally visible as oval defects on the material surface. Fig. 1 shows the surface of one material. The randomly distributed macroscopic defects are clearly visible. The size of these defects varies from a few $\mu \mathrm{m}$ up to $40 \mu \mathrm{m}$. In Fig. 1, the magnification of an approximate oval defect illustrates that macroscopic growth defects cause holes. Ga droplets are sketched as red ovals in the layer structure shown in Fig. 1. The formation of $\mathrm{Ga}$ droplets occurs anywhere in the material but not every Ga droplet leads to an oval defect on the material surface.

The layer schema also indicates that the examined 2DEG is realized in a heterojunction $320 \mathrm{~nm}$ beneath the surface at the GaAs-AlGaAs interface. The distance to the Si doping layer is about $70 \mathrm{~nm}$. Various specimens were structured under comparable conditions for every considered material. The structured specimens are Hall bars with a width of $0.2 \mathrm{~mm}$. Each Hall bar has a total length of $1.9 \mathrm{~mm}$, and the distance between two longitudinal ohmic contacts is about $0.3 \mathrm{~mm}$. The Hall bars were defined by photolithography and wet chemical etching. The magnetotransport measurements were performed in a dilution refrigerator with a base temperature of $T_{B}$ $=20 \mathrm{mK}$ and using low-frequency $(13 \mathrm{~Hz})$ lock-in technique.

In the following, we show how macroscopic growth defects seen as oval defects on the material surface influence the magnetotransport measurements. Therefore, we count the number of oval defects on the surface for each material. The density of oval defects increases with decreasing temperature at the Ga crucible orifice. Afterwards, we analyze each material by magnetotransport measurements at low temperatures $(T<1 \mathrm{~K})$ and determine the electron density and the electron mobility. Our first findings are presented in Fig. 2. Pictures of the material surfaces and measurements at low temperatures ( $T \sim 40 \mathrm{mK}$ ) of the longitudinal resistance $R_{x x}$ vs. magnetic field $B$ are shown for each material with the corresponding temperature at the $\mathrm{Ga}$ crucible orifice. The density of oval defects increases from Figs. 2(a)-2(c). Accordingly, Fig. 2(a) presents our observation for the highest orifice temperature. The corresponding picture of the material surface shows only a few oval defects $n_{S}($ Surface $)=675 \mathrm{~cm}^{-2}$, so we expect a negligible contribution of additional scattering events to the electron mobility. Astonishingly, the resulting electron mobility is with $\mu_{e}=0.5 \times 10^{6} \mathrm{~cm}^{2} / \mathrm{V}$ s low, and regarding the longitudinal resistance $R_{x x}$, the integer quantum Hall is barely developed. Furthermore, the quantum Hall effect collapses beyond the even filling factor $\nu=2$. The situation is completely different for an orifice temperature of $1000^{\circ} \mathrm{C}$ when the number of oval defects increases to $\mathrm{n}_{\mathrm{S}}$ (Surface) $=4750 \mathrm{~cm}^{-2}$ as presented in Fig. 2(b). Not only the corresponding electron mobility is higher $\left(\mu_{e}=4.4 \times 10^{6} \mathrm{~cm}^{2} / \mathrm{V} \mathrm{s}\right)$ but also features of the FQHE are seen in the longitudinal resistance $R_{x x}$ around $B=9.3 \mathrm{~T}$. In addition to the mobility also the electron density rises. By a further reduction of the orifice temperature and a simultaneous increase of the number of oval defects $\left(n_{S}(\right.$ Surface $\left.)=9250 \mathrm{~cm}^{-2}\right)$, the mobility $\left(\mu_{e}=6.2 \times 10^{6} \mathrm{~cm}^{2} / \mathrm{V} \mathrm{s}\right)$ and the electron density rise again as seen in Fig. 2(c). In the longitudinal resistance $R_{x x}$, we find even more fractional filling factors, e.g., $\nu=3 / 7$ at (a)

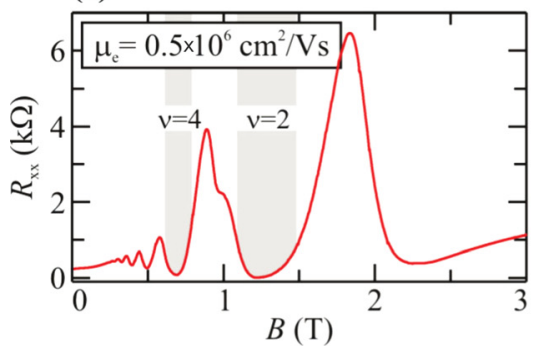

(b)

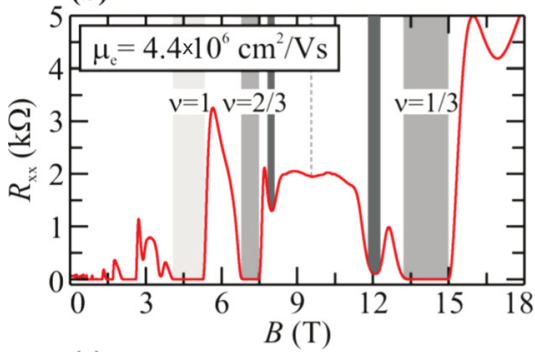

(c)

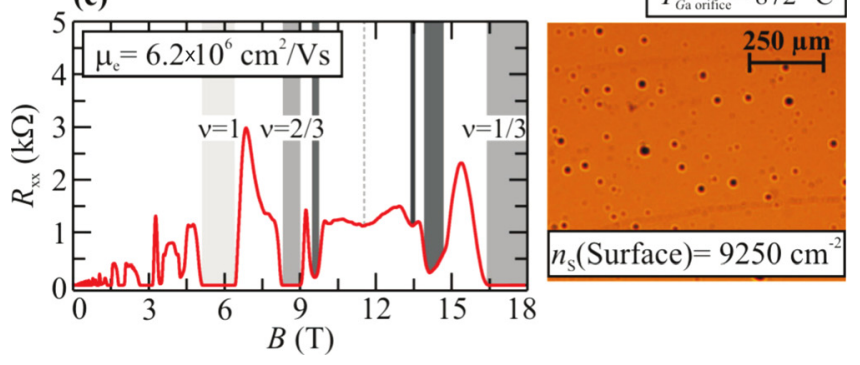

FIG. 2. Magnetotransport measurements were done over wide magnetic field range at $T \sim 40 \mathrm{mK}$. Here, the longitudinal resistance $R_{x x}$ is shown vs. magnetic field $B$ with a picture of the considered material surface. The number of oval defects rises from (a) to (c).

$B=13.5 \mathrm{~T}$ than in Fig. 2(b). Paradoxically, the material with the largest number of oval defects has also the highest electron mobility. This raises the question whether oval defects affect the 2DEG at all.

To prove the influence of oval defects on high-mobility 2DEGs, we examine an effect which is induced by rare strong scatterers. ${ }^{13,14}$ In high-mobility 2DEGs, negative magnetoresistances are usually observed around zero magnetic field. ${ }^{7,15-19}$ These negative magnetoresistances normally consist of a temperature independent peak around zero magnetic field and a temperature dependent huge magnetoresistance at larger magnetic fields both with a parabolic magnetic field dependence. ${ }^{15}$ A shoulder in the longitudinal resistivity marks the crossover between these different effects. Here, the examined negative magnetoresistance of each considered material is shown in Figs. 3(a)-3(c). The blue dot at the magnetic field $B_{c}$ corresponds to the crossover between the temperature independent section and the temperature dependent section. ${ }^{7,15}$ Recently, Ref. 7 discussed the origin of the peak and showed that it is caused by an interplay of long-range disorder potentials and rare strong scatterers. ${ }^{13,14}$ Therefore, the negative magnetoresistance around zero magnetic field can be expressed by ${ }^{7,13}$

$$
\rho_{x x}=\rho_{0}-\rho_{0} \frac{\omega_{c}^{2}}{2 \pi n_{S} v_{F}^{2}} f(x),
$$


(a) $\mu=0.5 \times 10^{6} \mathrm{~cm}^{2} / \mathrm{Vs}$
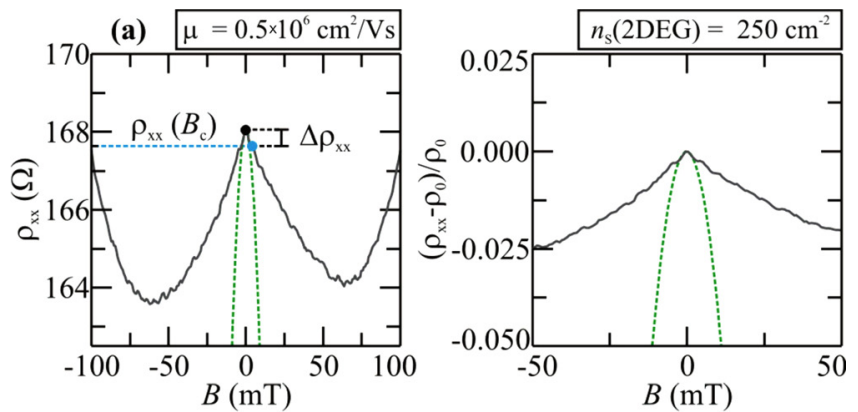

(b) $\mu=4.4 \times 10^{6} \mathrm{~cm}^{2} / \mathrm{Vs}$
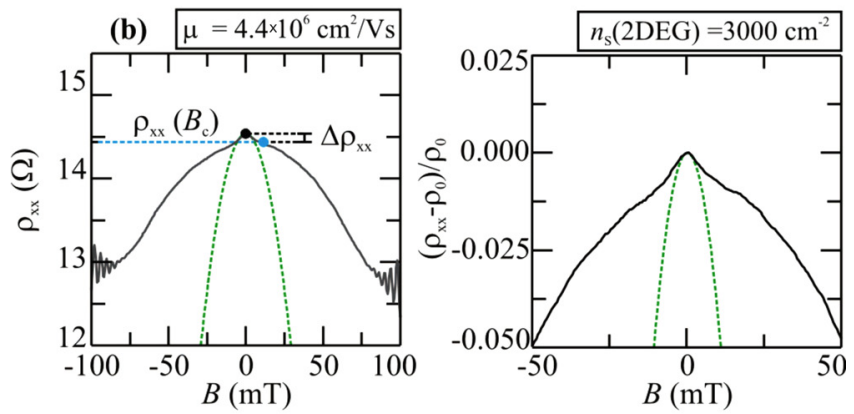

(c) $\mu=6.2 \times 10^{6} \mathrm{~cm}^{2} / \mathrm{Vs}$
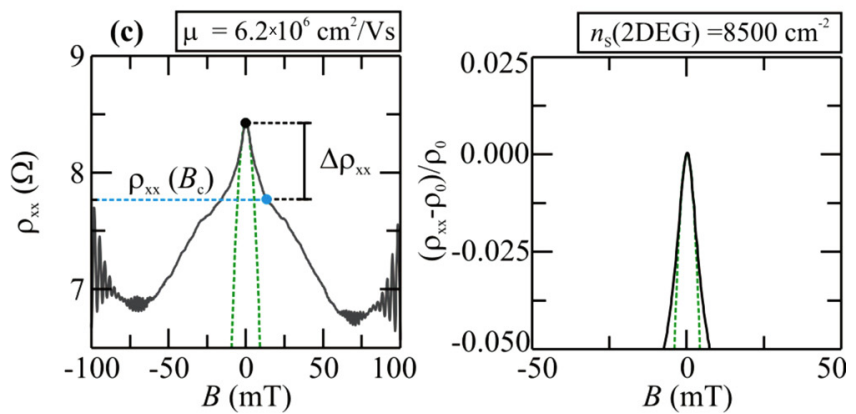

FIG. 3. The longitudinal resistivity $\rho_{x x}$ is shown vs. magnetic field $B$ for the three different heterostructures (a) - (c). The temperature independent section of the negative magnetoresistance around zero magnetic field is fitted by a parabola. A blue dot marks the crossover magnetic field $B_{c}$. The density of macroscopic growth defects is extracted from the negative magnetoresistance. Additionally, the normalized resistivity $\left(\rho_{x x}-\rho_{0}\right) / \rho_{0}$ is plotted vs. magnetic field to clarify that the negative magnetoresistance becomes more pronounced with the number of oval defects and the corresponding curvature increases.

where $\omega_{c}=e B / m^{*}$ is the cyclotron frequency, $v_{F}$ is the Fermi velocity, and $f(x)$ is given by

$$
f(x)=\frac{2}{x+1} \int_{0}^{\infty} d q \frac{q J_{1}^{2}(q)}{x q^{2}+2\left[1-J_{0}^{2}(q)\right]}
$$

with $x=\rho_{x x}\left(B_{c}\right) / \Delta \rho_{x x}$, and $J_{0,1}(q)$ are Bessel functions. The only unknown parameter in Equation (1) is the density of the rare strong scatterers $n_{S}$ which were identified as macroscopic growth defects. On the basis of the height of the temperature independent negative magnetoresistance $\Delta \rho_{x x}$ and

its curvature, the density of macroscopic growth defects $n_{S}$ next to $2 \mathrm{DEG}$ can be determined.

Considering Equation (1), we calculate the density of macroscopic defects $n_{S}(2 \mathrm{DEG})$ which influences the $2 \mathrm{DEG}$ for the samples discussed here. Therefore, we analyze the negative magnetoresistance at small magnetic fields under the same conditions for each material. We identify the temperature independent section with the help of temperature dependent magnetotransport measurements. Afterwards, the curvature of the negative magnetoresistance is determined by a parabola fitted to the experimental data, and the height is given by the difference between the longitudinal resistivity at zero magnetic field and the value at the crossover magnetic field $\rho_{x x}\left(B_{c}\right){ }^{7}$ Our results are summarized in Fig. 3. Also, the normalized resistivity $\left(\rho_{x x}-\rho_{0}\right) / \rho_{0}$ is plotted vs. magnetic field to be able to compare the observed negative magnetoresistances. The determined curvature increases with oval defect density.

Fig. 3(a) shows the magnetoresistance for the lowest density of oval defects $n_{S}($ Surface $)=675 \mathrm{~cm}^{-2}$. Here, the onset of the $\mathrm{SdH}$ oscillations is beyond the considered magnetic field range. However, a negative magnetoresistance is seen around zero magnetic field, and the temperature independent section is fitted by the dotted parabola. As expected from the material surface (see Fig. 2(a)), we extract a comparable low number of macroscopic defects $n_{S}(2 \mathrm{DEG})=250 \mathrm{~cm}^{-2}$. The value $n_{S}$ (surface) is higher than the value $n_{S}(2 \mathrm{DEG})$, indicating the smaller number of macroscopic defects acting as scattering center of the 2DEG. The corresponding normalized resistivity shows a small temperature independent negative magnetoresistance. With increasing number of oval defects, the negative magnetoresistance becomes more pronounced and also the SdH oscillations start at lower magnetic fields (see Fig. 3(b)). In this situation, $n_{S}(2 \mathrm{DEG})=3000 \mathrm{~cm}^{-2}$ macroscopic growth defects influence the 2DEG. The density of macroscopic defects $n_{S}$ is clearly different for an orifice temperature of $872{ }^{\circ} \mathrm{C}$ (see Fig. 2(c)). Correspondingly, we found here the highest density of macroscopic growth defects $n_{S}(2 \mathrm{DEG})=8500 \mathrm{~cm}^{-2}$.

Our results are summarized in Table I. One sees that the number of oval defects on the material surfaces $n_{S}$ (Surface) and the density of macroscopic growth defects $n_{S}$ (2DEG) determined from the negative magnetoresistance at small magnetic fields are comparable. Not only the number of macroscopic growth defects anywhere in the material increases by decreasing the temperature at the Ga crucible orifice, but also the carrier density rises showing, most likely, a decrease in the background impurity concentration and equivalently an increase of the mobility being much larger than expected from the increased carrier density. So, the mean free path is

TABLE I. Summary of our results with $\Lambda$ being the mean free path.

\begin{tabular}{|c|c|c|c|c|c|c|c|c|c|c|}
\hline $\begin{array}{l}T \\
\text { at Ga orifice } \\
\left({ }^{\circ} \mathrm{C}\right)\end{array}$ & $\begin{array}{c}n_{e} \\
\left(\mathrm{~cm}^{-2}\right)\end{array}$ & $\begin{array}{c}\mu_{e} \\
\left(\mathrm{~cm}^{2} / \mathrm{V} \mathrm{s}\right)\end{array}$ & $\begin{array}{c}\tau \\
(\mathrm{ns})\end{array}$ & $\begin{array}{c}\tau_{q} \\
(\mathrm{ps})\end{array}$ & $\tau / \tau_{q}$ & $\Delta \rho_{x x} / \rho_{0}$ & $\begin{array}{c}\Lambda \\
\mu \mathrm{m}\end{array}$ & $\begin{array}{c}n_{S} \\
\text { Surface } \\
\left(\mathrm{cm}^{-2}\right)\end{array}$ & 2DEG & $\begin{array}{c}\text { Distance } \\
(\mu \mathrm{m})\end{array}$ \\
\hline 1080 & $0.7 \times 10^{11}$ & $0.5 \times 10^{6}$ & 0.02 & 0.6 & 33 & 0.003 & 2 & 675 & 250 & 3400 \\
\hline 1000 & $1.1 \times 10^{11}$ & $4.4 \times 10^{6}$ & 0.15 & 1.6 & 94 & 0.009 & 22 & 4750 & 3000 & 190 \\
\hline 872 & $1.4 \times 10^{11}$ & $6.2 \times 10^{6}$ & 0.22 & 2.5 & 92 & 0.069 & 38 & 9250 & 8500 & 58 \\
\hline
\end{tabular}


larger for lower temperatures at the Ga crucible orifice. Despite the increasing number of macroscopic growth defects, the growth conditions seem to improve by lowering the temperature at the Ga crucible orifice. This assumption is proven by the enhanced quality of the considered 2DEGs which is reflected by an increase of the quantum relaxation time $\tau_{q}{ }^{5}$ This fact is also confirmed by the observation of the FQHE (see Fig. 2).

The quality differences between high-mobility 2DEGs can be determined in more detail by regarding the negative magnetoresistance at small magnetic fields. The higher quality of the third material is reflected by the well-developed two contributions of the negative magnetoresistance (see Fig. 3(c)). Here, the temperature independent section around zero magnetic field is clearly observable. Regarding the ratio of $\Delta \rho_{x x} / \rho_{0}$, one sees that it increases with oval defect density. For the highest mobility material, the contribution of the macroscopic growth defects to the zero field mobility $\mu$ is $6.9 \%$, i.e., a really sizeable contribution. The mean free path $\Lambda \approx 38 \mu \mathrm{m}$ of this material is comparable to the mean distance between macroscopic defects $(58 \mu \mathrm{m})$ which clearly shows the importance of this scattering mechanism.

Rare macroscopic growth defects next to high-mobility 2DEGs cause a sizable reduction of the mobility and a negative magnetoresistance. We found that the number of oval defects on the material surface reflects the density of macroscopic growth defects determined from the negative magnetoresistance around zero magnetic field. Paradoxically, growth conditions to achieve the highest mobility enhance also the number of oval defects.
We would like to thank A. W. Heine for help with the experiments. This work was financially supported by the Cluster of Excellence QUEST.

${ }^{1}$ D. C. Tsui, H. L. Stormer, and A. C. Gossard, Phys. Rev. Lett. 48, 1559 (1982).

${ }^{2}$ R. B. Laughlin, Phys. Rev. Lett. 50, 1395 (1983).

${ }^{3}$ R. Willett, J. P. Eisenstein, H. L. Stormer, D. C. Tsui, A. C. Gossard, and J. H. English, Phys. Rev. Lett. 59, 1776 (1987).

${ }^{4}$ V. Umansky, R. de-Picciotto, and M. Heiblum, Appl. Phys. Lett. 71, 683 (1997).

${ }^{5}$ S. Das Sarma and E. H. Hwang, Phys. Rev. B 90, 035425 (2014).

${ }^{6}$ P. T. Coleridge, R. Stoner, and R. Fletcher, Phys. Rev. B 39, 1120 (1989).

${ }^{7}$ L. Bockhorn, I. V. Gornyi, D. Schuh, C. Reichl, W. Wegscheider, and R. J. Haug, Phys. Rev. B 90, 165434 (2014).

${ }^{8}$ Y. G. Chai and R. Chow, Appl. Phys. Lett. 38, 796 (1981).

${ }^{9}$ G. D. Pettit, J. M. Woodall, S. L. Wright, P. D. Kirchner, and J. L. Freeouf, J. Vac. Sci. Technol. B 2, 241 (1984).

${ }^{10}$ K. Akimoto, M. Dohsen, M. Arai, and N. Watanabe, J. Cryst. Growth 73, 117 (1985).

${ }^{11}$ S.-L. Weng, Appl. Phys. Lett. 49, 345 (1986).

${ }^{12}$ M. Shinohara and T. Ito, J. Appl. Phys. 65, 4260 (1989).

${ }^{13}$ A. D. Mirlin, D. G. Polyakov, F. Evers, and P. Wölfle, Phys. Rev. Lett. 87, 126805 (2001).

${ }^{14}$ D. G. Polyakov, F. Evers, A. D. Mirlin, and P. Wölfle, Phys. Rev. B 64, 205306 (2001).

${ }^{15}$ L. Bockhorn, P. Barthold, D. Schuh, W. Wegscheider, and R. J. Haug, Phys. Rev. B 83, 113301 (2011).

${ }^{16}$ L. Bockhorn, A. Hodaei, D. Schuh, W. Wegscheider, and R. J. Haug, J. Phys.: Conf. Ser. 456, 012003 (2013).

${ }^{17}$ Y. Dai, R. R. Du, L. N. Pfeiffer, and K. W. West, Phys. Rev. Lett. 105, 246802 (2010).

${ }^{18}$ A. T. Hatke, M. A. Zudov, J. L. Reno, L. N. Pfeiffer, and K. W. West, Phys. Rev. B 85, 081304 (2012).

${ }^{19}$ R. G. Mani, A. Kriisa, and W. Wegscheider, Sci. Rep. 3, 2747 (2013). 